\title{
Use of Smartphone for Supportive Supervision in Nigeria
}

\author{
0. Onuka1, 0. Madukwe1, N. Chukwueme², M. Gidado33, U. Emperor ${ }^{2}$, K. Agbaiyero4, \\ 0. Adeleke ${ }^{5}$ \\ ${ }^{1}$ Abia State Tuberculosis Programme, Umuahia, Nigeria \\ ${ }^{2}$ National Tuberculosis and Leprosy Control Programme, Abuja, Nigeria \\ ${ }^{3}$ KNCV-TB CARE 1, Umuahia, Nigeria \\ ${ }^{4}$ National Tuberculosis and Leprosy Training Centre, Zaria, Nigeria \\ ${ }^{5}$ HS 2020-Abt Associate, Abuja, Nigeria \\ Email: onphil200@yahoo.co.uk
}

Received 7 April 2015; accepted 7 May 2015; published 12 May 2015

Copyright (C) 2015 by authors and Scientific Research Publishing Inc.

This work is licensed under the Creative Commons Attribution International License (CC BY). http://creativecommons.org/licenses/by/4.0/

(c) () Op Och Access

\section{Abstract}

Background: Based on in-country consultations, capacity to provide high-quality TB/TBHIV services is sub-optimal and not unrelated to weak supervision. Within the National TB Program (NTP) in Nigeria, supervision is entirely paper based; time consuming and structured in a way that rapid review of results is neither available nor timely resolution of problems. Objective: Describe the value of smart phones in improving quality of supervision. Methods: This is a descriptive study. With the NTP open to exploring innovative ways of strengthening supervision, Health Systems $20 / 20$ in collaboration with the NTP proposed a pilot use of smart phones for supervision at health facilities, with focus in Abia state. The State was purposively selected for the study. A stakeholders meeting was convened to fully engage all key TB partners. Situation analysis was conducted in Abia state, a south-east state selected for the pilot, out of which 4 facilities were identified. National and state supervisory checklists were reviewed, programmed $\&$ uploaded unto the smart phones. The State TB teams were trained on the use of the smart phone $\& 6$ supervisory visits were conducted within 4 consecutive quarters in Abia state for each facility. Results: Within the pilot sites, the smart phones were noted to be user friendly with rapid review of results done at each visit. Immediate feedback and corrective action for identified challenges were made. However, the record of previous findings and recommendations could not be retrieved on the spot during subsequent visits due to storage capacity of the phone. The time spent on supervision was reduced to 2 hrs per facility using the smart phones compared to $4 \mathrm{hrs}$ when using the paper based at other sites. With scale up from 4 to 45 DOT centers, the treatment success rate in the state TB program increased from $76 \%$ in 2011 to $85 \%$ in 2012 reporting year, for cases registered in the previous year. Conclusions: NTP through support of other partners should adopt and scale up the use of smart phones for supervision nationwide. 


\section{Keywords}

\section{Smartphone, Supervision}

\section{Introduction}

Tuberculosis is still a public health challenge especially in low income countries. Nigeria is ranked $4^{\text {th }}$ among 22 high burden countries [1]. Based on in-country consultations, capacity to provide high-quality TB/TBHIV services was sub-optimal and not unrelated to weak supervision by Tuberculosis control program managers. This is a reflection of low capacity of control program managers. However, human resource management is a vital and integral part of health system strengthening. "Human capital is the most important strategic resources for any organization" [2]. The complexity of public health system demands a strategic approach to human resource development. The quality of human resources is pre-requisites and vital to achieving sustained organizational performance; this was coined as "organizational performance is a function of these components: selecting the right people for the job, appraising their performance; rewarding them for good performance and developing their skills to improve performance in the future" [3].

There are different approaches in human capacity building which include long term in service training, short term training and supportive supervision. However supportive supervision is more cost effective way of improving the staff capacity and efficiency. Supportive supervision is a process of helping staff to improve their own work performance continuously (WHO 2013). Supportive supervision encourages open, two-way communication, and building team approaches that facilitate problem-solving. There are three supervision models, according to [1], categorized into autocratic, democratic and lassie' fare method. Autocratic method relies on formal authority, threats, pressure and close control. It is appropriate when task structure is high, employees need direction and quick results are required but often lead to resentment. Democratic method allows employees to influence and share in organizational decision making, relies on the supervisor conveying to employees what, how and why something is to be accomplished while lassie 'fare, delegates virtually all authority to employees to decide and act without the supervisor's involvement. This type of supervision reduces efficiency and affects productivity.

Supervision focuses on monitoring performance towards goals, and using data for decision-making, and depends upon regular follow-up with staff to ensure that new tasks are being implemented correctly. However the current method of supportive supervision has not yielded the needed result and requires a holistic and comprehensive fine-tuning to improve quality of care.

Within the National TB Program (NTP) in Nigeria, supervision is entirely paper based; time consuming and structured in a way that rapid review of results is neither available nor timely resolution of problems. With the NTP open to exploring innovative ways of strengthening supervision, decided to introduce electronic tool in supervision. A paradigm shift from paper based questionnaire to improved electronic documentation and reporting. This was succinctly coined as "Supervisors have indicated that the system is enabling them to monitor and assess performance of the TB health delivery system, identify problems and opportunities, and in many cases take immediate action for improvements" [4].

This does not replace the basic principles and process of supervision but tends to improve the efficiency of the process. The tool is expected to automatically generate reports at the end of each supervision process. This was tersely coined as "the automated checklist allowed for on the spot calculation and analysis, resulting in the improvement of supervision, assessment and creation of action plans" [5].

It is on this premise that the NTP proposed a pilot on use of smart phones for supervision at health facilities, with focus in Abia state. The essence of the supervision is to ensure effective care and improved quality of program performance in line with global target. The global and national TB target is to detect at least $70 \%$ of TB cases and achieve $85 \%$ treatment success rate.

\subsection{Objective}

1) Describe the value of smart phones in improving quality of supervision.

2) To ascertain the complementary effect of the tool on program performance. 


\subsection{Methodology}

This is a descriptive study with qualitative and quantitative arm. With the NTP open to exploring innovative ways of strengthening supervision, Health Systems 20/20 in collaboration with the NTP proposed a pilot use of smart phones for supervision at health facilities, with focus in Abia state. Abia state is in south eastern part of Nigeria and occupies 5834 square kilometer with a population of 3.9 million according to 2006 population. English is widely spoken is widely and serves as official language. They are majorly Christians. They are industrious, hospitable and accommodating.

A stakeholders meeting was convened to fully engage all key TB partners. Situation analysis was conducted in Abia state, a south-east state selected for the pilot, out of which 4 facilities were randomly selected. National and state supervisory checklists were reviewed, programmed \& uploaded unto the smart phones using the ODK software. The forms include the, Lab Direct Observation (used monthly), Laboratory Tool (Used monthly and quarterly, Specialized_TB_Lab_PCR (Used monthly and quarterly), TB_Clinic_Direct_Observation (Used monthly), TB_Clinic_ME_Tool (used quarterly) TB_Clinic_Tool (used monthly). “The automated checklist allowed for on the spot calculation and analysis, resulting in the improvement of supervision, assessment and creation of action plans" (Awa 2011).

The State TB teams were trained on the use of the smart phone and 6 supervisory visits were conducted within 4 consecutive quarters in Abia state for each facility. At the end of the supervisory visits, in-depth interviews were conducted for the supervisors and state control officers. The interviews were transcribed and analyzed.

There was desk review of state data to assess the complementary effect of the new tool on program quality, basically the treatment outcome. The favorable treatment outcomes are the cure rate and completion rate. The cured patients are those who completed their six month anti TB drugs and their six month Acid Fast bacilli (AFB) test result are negative while treatment completion are those who completed their six months treatment but could not do their six month AFB test. The treatment success rate is the summation of cure rate and treatment completion rate. The National TB control program adopted the WHO treatment success rate target of $85 \%$.

\subsection{Result of In-Depth Interview Analysis}

In explaining further on what supervision is, the in-depth interview participants were quick to point out that supervision is helping, guiding, and encouraging a staff to perform his task in line with set standards. Building the capacity of CV is an essential pre-requisite to enable them carry out their assigned tasks. From the discussions with the CVs, all were trained. The topics included overview of TB control program, supportive supervision, review of checklist and use of smartphone. It was attested during the interview that the training was adequate and beneficial to their assigned tasks and helped them perform effectively. This was succinctly put as I think the training was adequate and comprehensive (supervisor, in-depth interview 4). Analyzing the acceptance of the tool depicts that supervisors were elated with the new tool and showed willingness to use it. According to some supervisors, there were no technical hitches with the tool; they attested that the tool was user friendly. This was succinctly put as "the phone is similar to the smartphone we are using and easy to operate" (control officer in-depth interview 1 Appendix). In addition, a supervisor authenticated that "We were able to use the phone and complete the supervision" (supervisor in-depth interview 6).

Furthermore majority of the supervisors confirmed that the tool was user friendly. Timely generation of supervisory outcome and prompt resolution is vital and crucial for improving program performance. It was attested by the supervisors that there was automatic generation of supervisory outcome after completion of the checklist. It was interesting according to them to see the overall performance of the facility thereby providing a good opportunity for immediate feedback and timely resolution of challenges. This was tersely put as "the color indicators displayed the facility performance" (control officer in-depth interview 1) (Appendix).

Examining the time spent on supervision portrays that there was reduction of time spent on supervision from 4 hours using paper based questionnaire to 2 hour when the tool was used. Timely and appropriate feedback mechanism is crucial for effective program performance. It was revealed by in-depth interview that the tool automatically generated the outcome of the supervision, this provided a good opportunity for Immediate and timely feedback, rapid review of result as well as prompt resolution and corrective action for identified challenges were made. The supervision report is transmissible to state and national control program. With available internet and enhanced smartphone technology report transmission to higher level is easy and affordable. Also with dual 
sim card smartphones and improved network coverage report transmission to higher level is guaranteed. According to report of in-depth interview, there was no difficulty in sending the reports and they spend only five naira for the process. Despite all these successes, the supervisors had minimal challenges with the tool; according to them, retrieval of information of previous supervision was not possible and sending of result was difficult due to poor network service.

\section{Result}

Within the pilot sites, the smart phones were noted to be user friendly with rapid review of results done at each visit. Immediate feedback and corrective action for identified challenges were made. A record of previous findings and recommendations could not be retrieved on the spot during subsequent visits due to the capacity of the phone. The time spent on supervision was reduced to 2 hrs per facility using the smart phones compared to 4hrs when using the paper based at other sites. The TB direct clinic and laboratory observation checklist provided a good platform for mentoring of the supervisee. With scale up the use of smartphone from 4 to 45 DOT centers in the state, the treatment success rate in the state TB program increased from $76 \%$ in 2011 to $85 \%$ in 2012 reporting year, for cases registered in the previous year, indicating improved programme quality (Table 1).

Table 1. The treatment success rate.

\begin{tabular}{cccccc}
\hline $\begin{array}{c}\text { The treatment } \\
\text { success rate is the } \\
\text { summation of cure } \\
\text { rate and treatment } \\
\text { completion rate }\end{array}$ & $\begin{array}{c}\text { Cure rate } \\
\text { in } 2011\end{array}$ & $\begin{array}{c}\text { Completion rate in } \\
2011\end{array}$ & $\begin{array}{c}\text { Treatment success } \\
\text { rate in } 2011\end{array}$ & $\begin{array}{c}\text { Cure rate in } \\
2012\end{array}$ & $\begin{array}{c}\text { Completion rate in } \\
2012\end{array}$ \\
$\begin{array}{c}\text { Cure rate }+ \\
\text { completion rate }\end{array}$ & $76 \%$ & $0 \%$ & $76 \%$ & $84.07 \%$ & $1.00 \%$ \\
\hline
\end{tabular}

\section{Conclusion}

NTP through support of other partners should adopt and scale up the use of smart phones for supervision nationwide.

\section{References}

[1] NTBLCP Strategic Plan (2010-2015) Federal Ministry of Health, Abuja.

[2] Abdulmonem, A. and Rajesh, S. (2014) Impact of Human Resource Management Practice and Perceived Organizational Support on Job Satisfaction: Evidenced from Yanbu Industrial City, KSA. IUP Journal of Organizational Behavior, 13, 33-52.

[3] Goodwin, N. and Reinhold, G. (2006) Managing Health Services, Understanding Public Health Series, Maiden Head; Open University Press. Bull World Health Organization, 91, 602-611.

[4] Ekbladh, L. (2012) Smartphone for Supportive Supervision for TB. http//www.who.nt/ehealth

[5] Awa, D. (2011) Supportive Supervision (SS) for TB in Nigeria. Exerpt from Washinton DC Health System 20/20, Abt.Associates Inc. 


\section{Appendix}

\section{In-Depth Interview Guide}

In-Depth Interview Guide

1. What is your name?

2. How Long have you being working in TB unit?

3. What do you understand by supportive?

4. Have you had any training on supportive supervision?

5. How is the new tool?

6. The training on the use of the new tool was it adequate and sufficient?

7. Did you experience any challenge using the tool

8. Did the tool generate result as expected?

9. What is the average time for supervision?

10. Has the tool helped to improved your supervision efficiency?

11. Did you experience challenge in transmitting the result to next level

12. How was the report of the supervision resolved?

13. Do you think that the tool should be scaled up?

\section{In-Depth Interview Analysis}

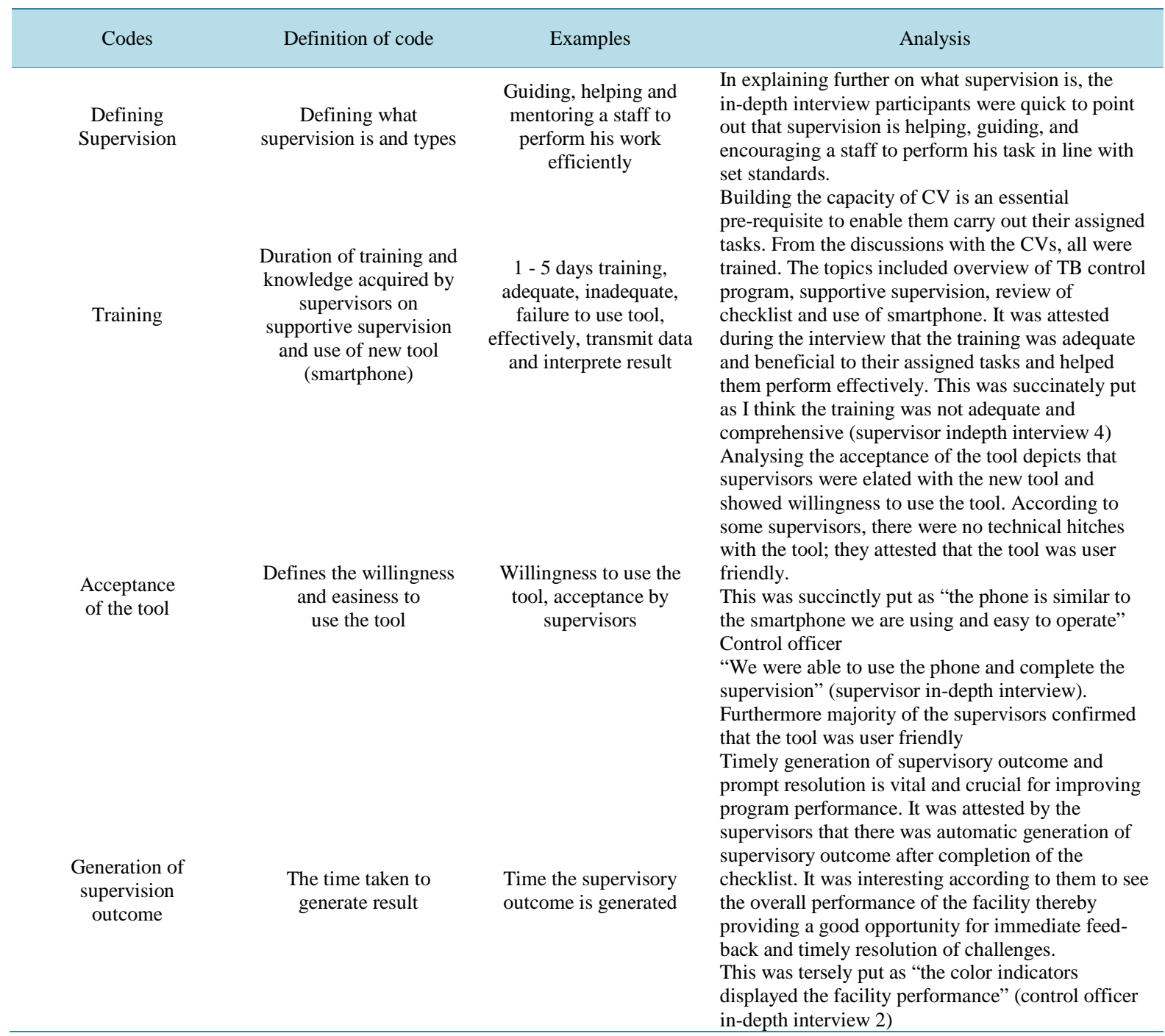




\section{Continued}

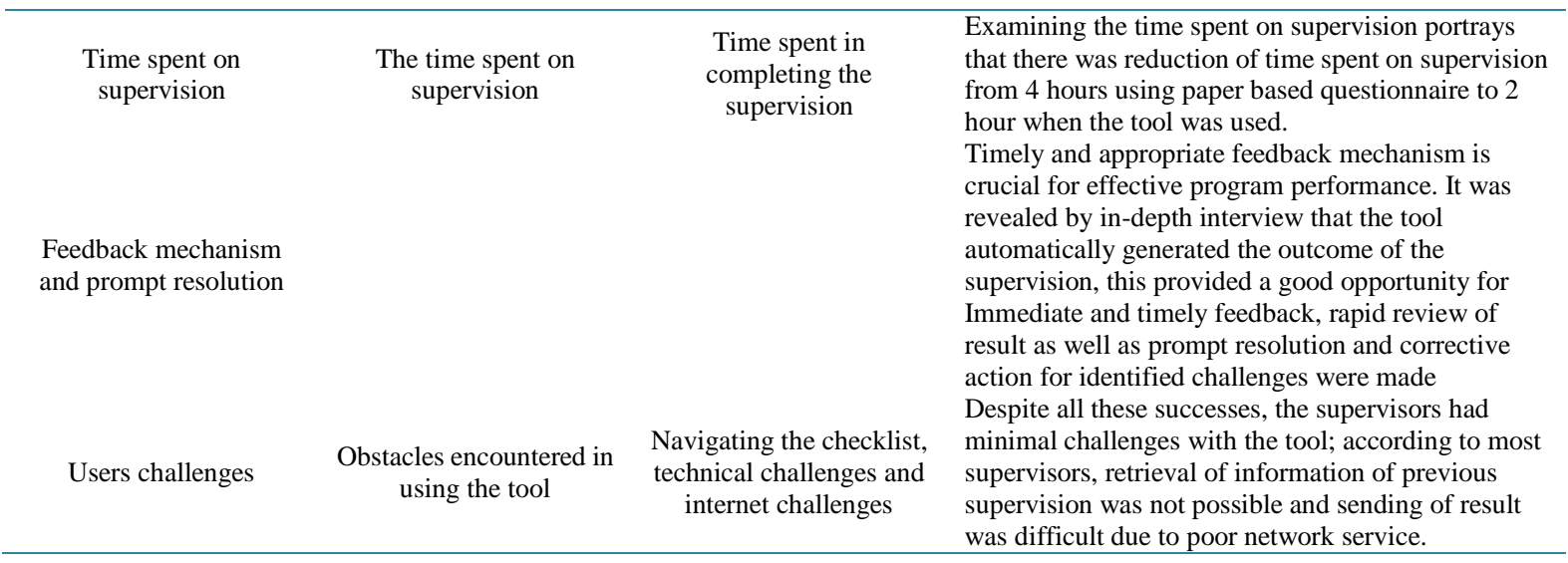

